between AS pts and HC (10.5; 8.3-18.0 pg/ml vs 11.9; 8.2-18.3 pg/ml, p>0.05). The same levels of IL-6 and IL-8 were detected in AS with IBD and AS without signs of IBD $(p>0.05)$. In AS pts, serum IL-6 concentration was positively correlated with ASDAS $\operatorname{ESR}(r=0.3)$, ASDAS CRP $(r=0.3)$, ESR $(r=0.3)$ and CRP $(r=0.5)(p<0.05)$; IL-8 was negatively associated with presence of fecal calprotectin $(r=-0.3)(p<0.05)$. Conclusion: Elevated serum concentration of IL-6 in AS is associated with clinical and laboratory markers of high inflammatory activity of the disease. The levels of IL-8 in the sera of AS patients were negatively correlated with the concentration of fecal calprotectin. Data on the relationship of IL-8 with the activity of the pathological process in AS require further study.

Disclosure of Interests: Elena Aleksandrova: None declared, Alexander Novikov: None declared, Polina Kulakova: None declared, Aleksey Dorofeev: None declared, Nadezhda Savenkova: None declared, Evgeniy Volnukhin: None declared, Anton Kovshik: None declared, Galina Lukina Speakers bureau: Novartis, Pfizer, UCB, Abbvie, Biocad, MSD, Roche DOI: 10.1136/annrheumdis-2020-eular.3082

\section{FRI0565 $\quad$ PREVALENCE AND SIGNIFICANCE OF ANTIBODIES AGAINST CITRULLINATED ALPHA-ENOLASE (ANTI- CEP1) IN CONNECTIVE TISSUE DISEASES.}

A. Alunno ${ }^{1}$, F. Carubbi ${ }^{2}$, O. Bistoni ${ }^{1}$, M. Antonucci ${ }^{1}$, E. Bartoloni Bocci ${ }^{1}$, R. Giacomelli', R. Gerli1. ${ }^{1}$ Rheumatology Unit, University of Perugia, Perugia, Italy; ${ }^{2}$ Rheumatology Unit, University of L'Aquila, LAquila, Italy

Background: Anti-cyclic citrullinated peptide (anti-CCP) auto-antibodies represent the current gold standard for the diagnosis of rheumatoid arthritis (RA). However, growing evidence suggests that a variety of other citrullinated self-proteins may act as autoantigens and lead to the production of autoantibodies (1). Furthermore, autoantibodies believed to be RA-specific have been detected also in patients with connective tissue diseases (CTDs). We recently demonstrated that antibodies against citrullinated alpha-enolase (anti-CEP1) are a biomarker of erosive disease and RA-associated interstitial lung disease (2).

Objectives: The purpose of this study was to investigate the prevalence and possible prognostic value of anti-CEP-1 in patients with CTDs.

Methods: Two hundred and twelve consecutive patients with CTDs (51 systemic lupus erythematosus (SLE), 85 primary Sjogren's syndrome (pSS) and 76 systemic sclerosis (SSc)) were studied and compared to 97 sex and age matched normal controls (NC) and 267 patients with RA. Anti-CEP1 IgG were detected in serum samples with a commercial ELISA kit (Euroimmun).

Results: The overall prevalence of anti-CEP1 in CTDs was 7\% (15/212 patients). In detail, these antibodies were detectable in 4 out of 85 pSS (5\%), 5 out of 51 SLE $(10 \%)$ and $6 / 76 \mathrm{SSc}(8 \%)$. The prevalence and the titer of anti-CEP1 in CTDs was significantly higher compared to NC and significantly lower compared to RA. Anti-CEP1 positive patients did not display a specific clinical and serological picture. Unlike in RA, anti-CEP1 did not correlate with CTD-associated ILD.

Conclusion: This is the first study assessing anti-CEP1 in a large cohort of patients with CTDs. We demonstrated that the association of these autoantibodies with ILD is specific for RA since it is not observed in SLE, pSS and SSc. Furthermore, although being significantly more prevalent and at higher titer compared to $\mathrm{NC}$, anti-CEP1 do not allow to discriminate different patient subsets displaying peculiar clinical or serological phenotypes. Based on our results, the application of anti-CEP1 in CTDs is not advisable, however larger studies may possibly identify correlations not evident in our cohort.

References:

[1] Bonifacio AF, Alunno A, La Paglia GMC, Valentini E, Leone MC, BartoIoni E, Gerli R. Novel autoantibodies in rheumatoid arthritis. Reumatismo 2019;71(1):1-12

[2] Alunno A, Bistoni O, Pratesi F, La Paglia GMC, Puxeddu I, Migliorini P, Gerli R. Anti-citrullinated alpha enolase antibodies, interstitial lung disease and bone erosion in rheumatoid arthritis. Rheumatology (Oxford). 2018;57(5):850-855

Disclosure of Interests: Alessia Alunno: None declared, Francesco Carubbi Speakers bureau: Francesco Carubbi received speaker honoraria from Abbvie and Celgene outside this work., Onelia Bistoni: None declared, Matteo Antonucci: None declared, Elena Bartoloni Bocci: None declared, Roberto Giacomelli Grant/research support from: Actelion, Pfizer, Speakers bureau: Abbvie, Roche, Actelion, BMS, MSD, Ely Lilly, SOBI, Pfizer, Roberto Gerli: None declared DOI: 10.1136/annrheumdis-2020-eular.4549

\section{FRI0566 \\ THE FLARE-RA QUESTIONNAIRE CAN IDENTIFY OMERACT FLARES IN PATIENTS WITH RHEUMATOID ARTHRITIS INCLUDED IN THE TAPERA TRIAL}

D. Bertrand ${ }^{1}$, V. Stouten ${ }^{1}$, S. Pazmino ${ }^{1}$, D. De Cock ${ }^{1}$, A. Moeyersoons ${ }^{2}$, R. Westhovens ${ }^{1,2}$, J. Joly ${ }^{2}$, P. Verschueren ${ }^{1,2} .{ }^{1} \mathrm{KU}$ Leuven, Skeletal Biology and

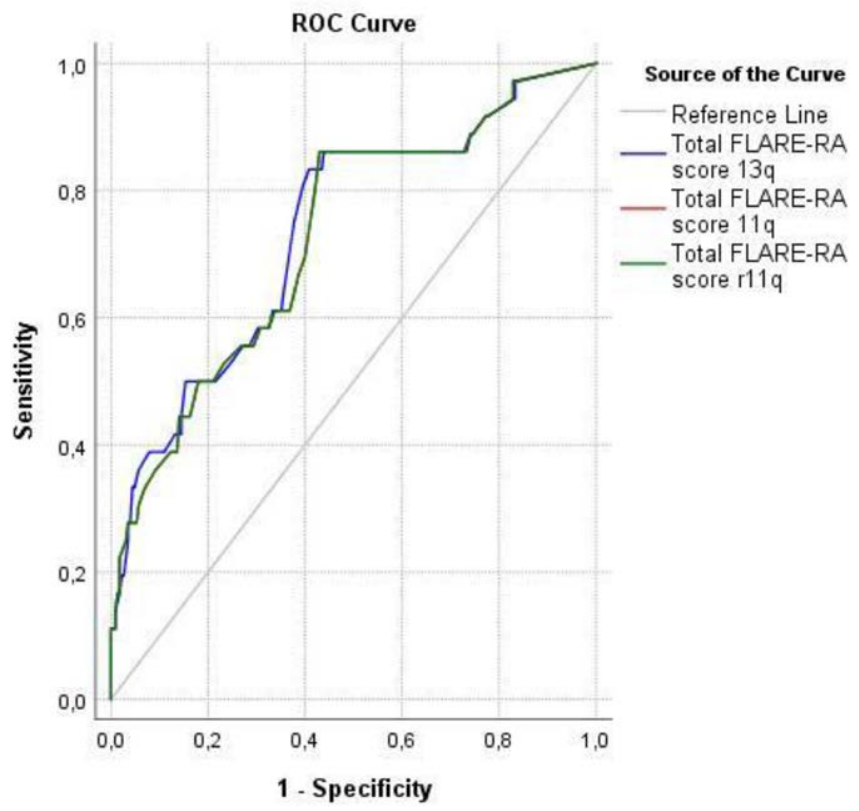

Figure 1. ROC curves of the total FLARE-RA scores (13q, 11q and r11q version) for identifying a flare according to the OMERACT definition.FLARE $Q$ is expressed in mean \pm SD. M month, FLARE Q FLARE-RA questionnaire 13q, n number, LDA low disease activity, MDA moderate disease activity, HDA high disease activity

Engineering Research Center, Leuven, Belgium; ${ }^{2}$ UZ Leuven, Rheumatology, Leuven, Belgium

Background: The Flare assessment in rheumatoid arthritis (FLARE-RA) questionnaire has been developed to identify flares in patients with rheumatoid arthritis (RA). The first version was published by Berthelot et al. (2012) and consisted of 13 questions on a Likert-scale of 1-6 ranging from 'completely untrue' to 'completely true.' When the FLARE-RA questionnaire was validated by Fautrel et al., 2 questions were removed, and it was rescaled to $0-10$. The questionnaires' usefulness has been tested in few studies. Further external validation in a well-defined cohort of patients with RA is needed.

Objectives: To externally validate the FLARE-RA questionnaire and determine cut-offs for identifying a flare in an established RA population in which biologicals are tapered.

Methods: Patients who were in remission according to the DAS28CRP or ESR ( $\geq 6$ months) and treated with etanercept $50 \mathrm{mg}$ weekly ( $\geq 1$ year) were enrolled between $2012-2014$ in the pragmatic 1-year open-label randomised controlled TapERA (Tapering Etanercept in RA) trial. Patients were randomised to continue etanercept $50 \mathrm{mg}$ weekly or taper to $50 \mathrm{mg}$ every other week. The FLARE-RA questionnaire (version of 2012) was completed every 3 months. Outcomes were based on 3 versions of the questionnaire (13 questions (13q), 11 questions (11q) and 11 questions rescaled $(r 11 q)$ ). Per time point, the average of the answers was calculated to obtain a total score of the FLARE-RA questionnaire. The total scores were compared between patients in remission (DAS28CRP $<2.6$ ), low (DAS28CRP $\geq 2.6$ $\leq 3.2$ ), moderate (DAS28CRP $>3.2-\leq 5.1$ ) and high disease activity (DAS28CRP >5.1) using the Kruskal-Wallis test and between patients with and without a flare according to the OMERACT definition (increase in DAS28 $>1.2$ compared to baseline or increase in DAS28 $>0.6$ and current DAS28 23.2) using the Mann-Whitney $U$ test. The total FLARE-RA scores of the different time points were combined to determine the receiver operating characteristics (ROC) curves, the corresponding cut-off values and the area under the curve (AUC) for identifying an OMERACT flare. An AUC of $<0.5$, between 0.5 and 0.7 and $>0.7$ stands for having no, moderate and a good predictive value, respectively.

Results: FLARE-RA questionnaires of 66 patients ( $68 \%$ female, mean \pm standard deviation (SD) age of $55 \pm 11$ years) were collected. The FLARE-RA score (13q) did increase when disease activity increased at month (M) 3 and M12 ( $p<0.01$ ) (table 1). Patients presenting with an OMERACT flare had a statistically significantly highe total FLARE-RA score (13q) compared to patients without a flare, except at M12 (M3 and M6: $p<0.05$, M9: $p<0.01$ ). The AUC - ROC curve of the FLARE-RA questionnaire (13q) for identifying an OMERACT flare was 0.736 and the cut-off value was 2.3 (1-6 scale). The AUC - ROC curve was the same for the 11q and r11q version, namely 0.727 . The cut-off values were 2.4 (1-6 scale) and 2.7 (0-10 scale), respectively (figure 1). 